\title{
WSNs Performance Evaluation Using Non-equilibrium Statistical Mechanics Method
}

\author{
Chen Ye \\ College of Science \& Technology, Ningbo University, Ninigbo, Zhejiang, China \\ yechen@nbu.edu.cn
}

\begin{abstract}
In recent years, with the further development and more applications emerging, WSNs is no longer confined to the traditional area. Because WSNs is dynamic, distribution, resource constrained, uncertainty, complexity and heterogeneity, meanwhile distinct WSNs applications have distinct characteristics and requirements in the respective field, which both urge us to further research, especially performance evaluation of WSNs. In this paper, we expand the range of performance index which exceeds time delay, packet loss rate, and throughput. Meanwhile, put forward a non-equilibrium statistical mechanics model for integrated WSNs performance evaluation from an evolutionary perspective. A non-linear information dynamics mode is introduced based on the maximum flux principle during the modeling process. The integrated performance evaluation of WSNs can be done by giving reasonable weights of each evaluation index to stabilize the system. The new model reveals the formation pattern and the dynamics law of evolution. The proposed method has been serviced in complex simulation, and the results have indicated that the model is objective and effective.
\end{abstract}

Keywords: WSNs, non-equilibrium statistical mechanics method, performance evaluation

\section{Introduction}

With the rapid development of embedded computing technology, modern network, wireless communication technology, wireless sensor network is popular in the world which integrates relative technology together [1-3]. The wireless sensor network has very vast application prospect and potential utility value, opens up a wealth of opportunities for distinct applications, such as agriculture, city management, environmental monitoring, health management, emergency rescue and disaster relief, anti-terrorism, remote control of hazardous area and so on [4-7]. WSNs are becoming the hottest research fields concerning in the international community, involving cross multidisciplinary and highly integrated knowledge [8-9]. With the further development and more applications emerging, WSNs is no longer confined to the traditional area, for example WSNs can currently operate heating, ventilating, and air-conditioning and lighting systems efficiently, moreover improve the comfort of occupants while reducing energy consumption, and additionally augment the safety and security systems. There have been several large-scale sensor network deployments reported during the past years [10-11], WSNs has been highly paid more attention by academic and industrial circles in many countries, and been regarded as one of most shining technology which has the huge influence on twenty-first Century [12-14].

There are a large number of researches on WSNs mainly concentrated in the protocol stack development, network topology design, algorithms in each protocol layer, application 
prospects and other aspects [15-18]. At present, there is no longer a unified standard and rank framework, according to the performance index of WSNs. In distinct application environment, the network performance of WSNS shown are very distinct, we must put forward an overall analysis and corresponding evaluation method for WSNs performance index of the corresponding. Gholami, N. Cai emphasized the temporal performance dynamics of wireless links and provided important findings about such phenomenon [19]. Okdem, Selcuk had proposed evaluating WSNs performance from a global or overall angle [20]; Bhuyan, Bhaskar presented a comprehensive study to quantify and characterize link quality [21]. Dohler, Mischa, et al. proposed a video fire detection system which can acquire and transfer multimedia data in various occasions, so the requirement of robustness and throughput for WSNs is higher [22]. Ravindranath, Lenin, et al. proposed a model to calculate the path performance evaluation value of WSNs, using the weighted average method [23]. WSNs must meet real time information acquisition requirements, such as gas concentration, personnel location, temperature and humidity parameter on the mine, so it has high level requirement of the network delay [24]. Zhang, Vijay Varadharajan evaluated WSNs performance binding relative comparison method and variation coefficient method together [25]. Padmavathi, G., D. Shanmugapriya investigated radio interference and point out the inaccuracy of range-based interference model [26]. Therefore, comprehensive performance of WSNs is very important and beneficial for planning and management of a construction network.

In current researches, there are few articles and no uniform standard about WSNs performance. Only some related researches for the parameters for WSNs performance, which are speed, accuracy, dependability, availability and so on [27-28]. On the one hand, WSNs performance indexes usually are very limited [29], which neglects the other factors which have huge effect on WSNs performance. On the other hand, indexes have influence on each other, meanwhile weight is ignored. Therefore, with considering the complex characters of WSNs in distinct applications, in this paper, firstly, we expand the range of performance index which includes accountability, agility, cost, actual performance, security and privacy. Secondly, we build a non-equilibrium statistical mechanics model for integrated WSNs performance evaluation from an evolutionary perspective. Finally, we apply Opnet network simulation software to collect simulation data of WSNs, and then we verify the proposed model, the evaluation results demonstrate us that it is efficient and effective.

\section{Evaluation Model based on Non-equilibrium Statistical Mechanics}

The competitive process of complex network system follows the maximum flow principle. An open complex system faraway from balance, always looks for an optimization process, to make system achieve maximum generalized flow under the given constraints or cost. WSNs system is a complex network system, the sharing formulation process of various typical influence factors(include accountability, agility, cost, actual performance, security and privacy.) is the competition process with each other, so the WSNs evolution system is in accordance with the principle of maximum flow.

Assuming the index property of sub-elements in WSNs is $x=\left(x_{1}, x_{2}, \cdots, x_{n}\right)$, while there is competition and cooperation among $x_{i} . x_{1}, x_{2}, \cdots, x_{n}$ respectively is the driving force of each subsystem evolution. All micro subsystems in gamma space $\Gamma$ form a continuous unit, and then $d x=\left(d x_{1}, d x_{2}, \cdots, d x_{n}\right)$ is a volume element of gamma space $\Gamma$. If the generalized flux is $J$ when the state of the system exists within the volume unit $d x$ at 
time $t$, then the averaged flux over all possible micro-states can be expressed as follows:

$$
\bar{J}=\int \rho(x, t) J(x) d x
$$

Among them, $\rho(x, t)$ is the time-varying probability density distribution function, the generalized flow function can be expressed as:

$$
J=\eta+\sum_{i} r_{i} x_{i}+\sum_{i j} r_{i j} x_{i} x_{j}+\sum_{i j k} r_{i j k} x_{i} x_{j} x_{k}+\sum_{i j k l} r_{i j k l} x_{i} x_{j} x_{k} x_{l}+\cdots
$$

Where $\eta$ and $r$ are constants, and $i, j, k, l$ are the numbers of elements. The remaining summation terms indicate the coupling effect between distinct components or indexes, $R$ as the coupling coefficient. The subsystem reflects the performance of WSNs system, its character is expressed by the interaction of each element under the generalized flow. The generalized flow's function must satisfy certain constraints, these constraints are that the components should meet certain conservation or the complex boundary reflecting the open characteristic, and it can be generalized to write to one to four order effect matrix form:

$$
\left\{\begin{array}{l}
f_{1}=\left\langle x_{i}\right\rangle \\
f_{2}=\left\langle x_{i} x_{j}\right\rangle \\
f_{3}=\left\langle x_{i} x_{j} x_{k}\right\rangle \\
f_{4}=\left\langle x_{i} x_{j} x_{k} x_{l}\right\rangle
\end{array}\right.
$$

According to the principle of maximum flow, given the constraint formula (3) we can achieve the maximum generalized flow by formula (1), use Lagrange optimization calculation, there can obtain the probability density function:

$$
\rho=c\left(J-\alpha-\sum_{i} \beta_{i} x_{i}-\sum_{i j} \beta_{i j} x_{i} x_{j}-\sum_{i j k} \beta_{i j k} x_{i} x_{j} x_{k}-\sum_{i j k l} \beta_{i j k l} x_{i} x_{j} x_{k} x_{l}\right)^{-1}
$$

In the formula, $c$ is the constant $\alpha$ and $\beta$ are the optimization parameters in Lagrange optimization. Put formula (2) into formula (4), we can get:

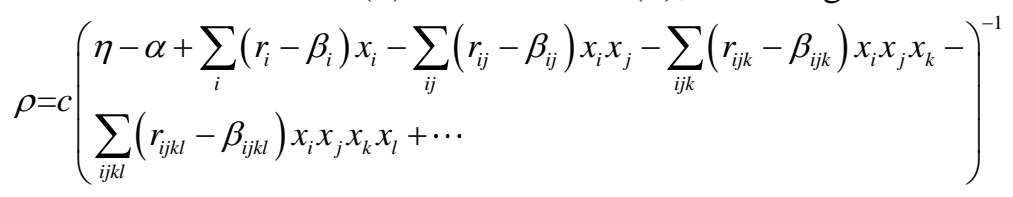

In formula (5) the parameters $\alpha$ and $\beta$ directly regulate the Micro dynamic rules of component interaction. According to Taylor, formula (5) can be unfolded as the following:

$$
\rho=\exp \left(\mu+\sum_{i} \sigma_{i} x_{i}+\sum_{i j} \sigma_{i j} x_{i} x_{j}+\sum_{i j k} \sigma_{i j k} x_{i} x_{j} x_{k}+\sum_{i j k l} \sigma_{i j k l} x_{i} x_{j} x_{k} x_{l}+\cdots\right)
$$

In the formula (6) the index items can be used as a potential function $\phi$, it can ensure the asymptotic stability of system reference state, effectively control the properties of structure of the complex evaluation subsystem. Here potential function $\phi_{\text {is defined as: }}$

$$
\phi(\sigma, x)=\mu+\sum_{i} \sigma_{i} x_{i}+\sum_{i j} \sigma_{i j} x_{i} x_{j}+\sum_{i j k} \sigma_{i j k} x_{i} x_{j} x_{k}+\sum_{i j k l} \sigma_{i j k l} x_{i} x_{j} x_{k} x_{l}+\cdots
$$


Give the potential function in formula (7), a parallel transformation and make the constant terms of transformation by a diagonalization of matrix, we can get:

$$
\xi_{j}=\sum_{i=1}^{n} a_{j i} x_{i}
$$

And the potential function turns into:

$$
\bar{\phi}(\lambda, \xi)=\zeta+\sum_{j} \lambda_{j} \xi_{j}^{2}+\cdots
$$

From formula (9) we can see, ${ }^{\xi_{j}}$ is the combination model of components' driving force $^{x_{i}}$, it reflects the entire possible structure model; it is similar to the order parameter. Based on the potential function and evolution equation of dynamic function, from (9) we can conclude the ordered structure evolution dynamic equation as:

$$
\dot{\xi}_{j}=\lambda_{j} \xi_{j}+S_{j}\left(\xi_{1}, \xi_{2}, \cdots, \xi_{n}\right)+F_{j}(t)
$$

(9) and (10) are the foundation of stability of all state patterns during the evolution of the WSNs system. The competition, coordination and other interactions (numerical value and plus sign or minus sign of ${ }^{a_{j i}}$ are employed to describe the relation and degree of interaction in the model) between internal elements, system elements form distinct composite patterns $\xi_{j}$ (corresponding to distinct values of $\lambda_{j}$ ) through distinct patterns of cooperation and competition in Figure 2.

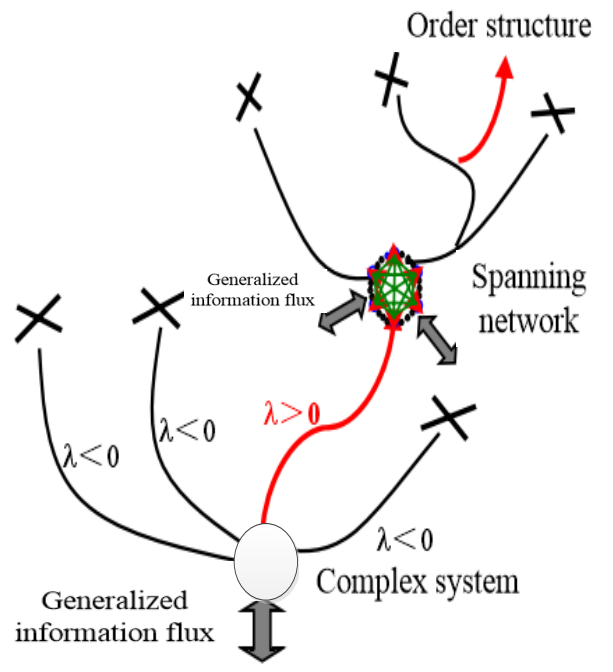

Figure 1. Evolutionary Model of WSNs System

Thus, in formula (10), when $\lambda_{j}>0$, it corresponds to active composite patterns. In other words, among these composite patterns, only the structure with the largest ${ }^{\lambda_{j}}$ is the most active, predominant and reasonable. Other potential patterns all belong to transient fast variables, which will be eliminated. The system is damped and can be eliminated when ${ }^{\lambda_{j}<0}$.The method which selects active and predominant patterns and eliminates driven patterns through competition based on corresponding control conditions 
constitutes the theoretical foundation of identifying and evaluating state patterns of WSNs performance. The method which choses the active and leading function model and generously abandons the driven model according to the control condition makes the theoretical basis of analyzing and evaluation of the WSNs performance.

\section{The Self-organizing Feature Mapping and Simulation for the New Evaluation Model}

Due to the characters of dynamic, distribution, resource constrained, uncertainty, complexity and heterogeneity, meanwhile distinct WSNs applications have distinct characteristics and requirements in the respective field, which both urge us to further research, especially performance evaluation of WSNs. we can apply non-equilibrium statistical mechanics for integrated WSNs performance evaluation from an evolution perspective. The principle of maximum flow for integrated performance assessment of WSNs system provides a method of pattern recognition. When the values of coefficients $\beta$ and $r$ are determined, the corresponding systematic structural pattern ${ }_{j}$ can be obtained. The competition characteristic of SOFM network algorithm in Figure 3 is the same to the MFP arithmetic.

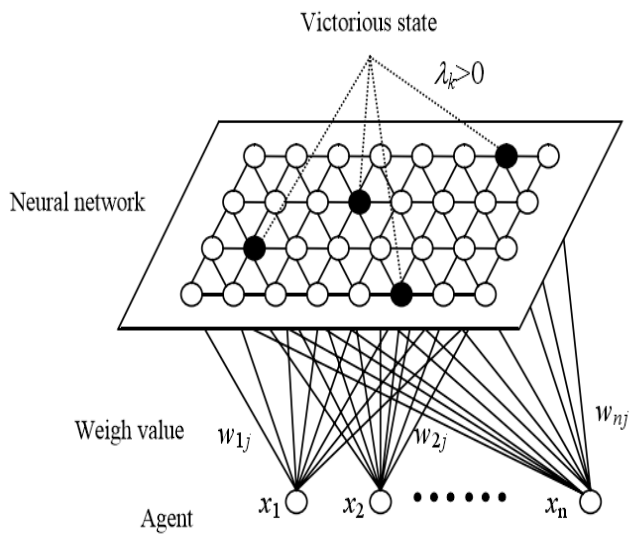

Figure 2. SOFM Illustration of Dynamic Simulation System

Neurons in distinct areas of the space have distinctive functions. The network has unique characteristics reacting to the external input information. The connecting weights between neurons can be obtained by self-organization training; thereby the characteristic property of the entire system can be determined. The connecting weight corresponds to ${ }^{j i}$ in formula (8). Pattern $\xi_{j}$ obtained by selecting certain parameters is just the result of competition and optimization of the system. Figure 3 displays the pattern of winning, which also needs recognition and evaluation. In a given neuron network, the number of nodes in input layer nodes is $m$, hidden layer node is $l$, output layer nodes is $g$. To facilitate the representation, we take it as input layer node, $k$ as hidden layer node, $p$ as output layer node. The neural network model is not different to appear provincial minimum, and has the disadvantage of slow convergence rate. Therefore we integrate non-equilibrium statistical mechanics which get 
the characteristic of global search and efficiency, with fuzzy optimization neural network algorithm together.

\section{A Numerical Example}

In this paper, we evaluate the performance of wireless sensor network based on ZigBee technology, using network simulation software OPNET to build distinct networks. Based on the simulated measurement, we collect network performance data, and evaluate the comprehensive performance of WSNs. To explore the relationships between these metrics, a series of tests were conducted with a single radio transmitting to a receiver, which will require low data-rate communications, hardware was selected that conformed to the IEEE 802.15.4 standard for low-rate wireless personal area networks.

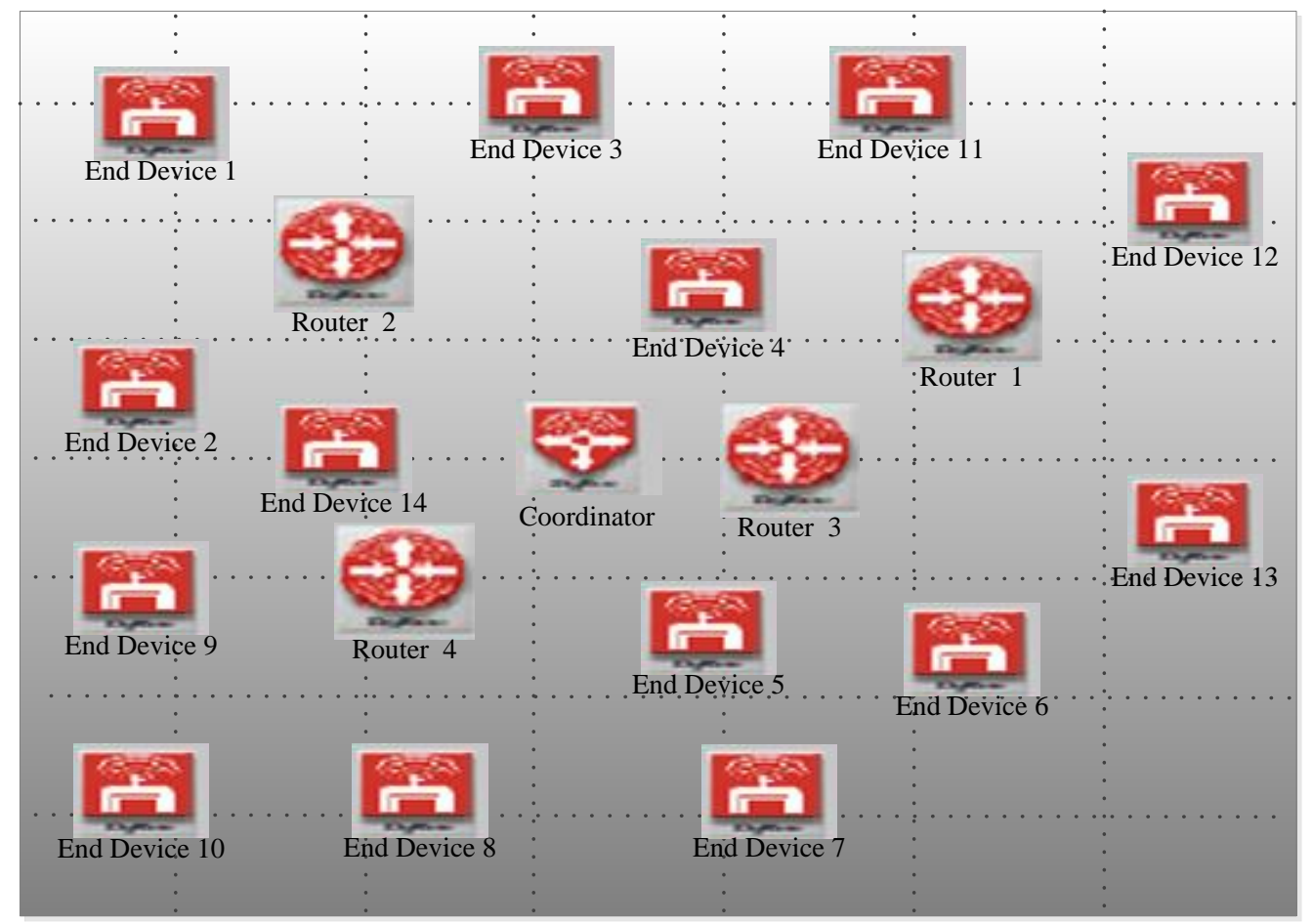

Figure 3. WSNs Simulation Environment

\subsection{WSN Simulation}

The WSN comprises of 1 network coordinator, 4 routers and 14 terminal nodes. Each terminal node and router can transmit and receive data packets, and can communicate with each other. In the simulation run time, randomly generate packets and send through the shortest path to the destination. Performance indicators selected for the network are accountability, agility, cost, actual performance, security and privacy.

These factors are classified into two types. Factors $x_{1}$ to $x_{4}$ belong to the type in which the bigger of the value the better. Factor $x_{5}$ belongs to the type in which the smaller of the value the better. The data of $10 \mathrm{WSNs}$ collected by simulation are shown in Table 1. 
Table 1. Statistical Practical Data of WSNs

\begin{tabular}{|c|c|c|c|c|c|c|}
\hline \multirow[b]{2}{*}{$\begin{array}{l}\text { Number of } \\
\text { WSNs }\end{array}$} & \multirow[b]{2}{*}{$\begin{array}{l}\text { Accountability } \\
\qquad\left(x_{I}\right)\end{array}$} & \multicolumn{2}{|c|}{ actual performance $\left(x_{2}\right)$} & \multirow[b]{2}{*}{$\operatorname{agility}\left(x_{3}\right)$} & \multirow[b]{2}{*}{$\begin{array}{c}\text { security } \\
\text { and } \\
\operatorname{privacy}\left(x_{4}\right)\end{array}$} & \multirow[b]{2}{*}{$\operatorname{cost}\left(x_{5}\right)$} \\
\hline & & $\begin{array}{c}\text { time } \\
\operatorname{delay}\left(x_{21}\right)\end{array}$ & $\begin{array}{c}\text { packet } \\
\text { loss } \\
\text { rate }\left(x_{22}\right) \\
\end{array}$ & & & \\
\hline 1 & 3 & 0.010150 & 0.013690 & 9.6 & 4 & 112 \\
\hline 2 & 4 & 0.070866 & 0.002560 & 8.7 & 5 & 120 \\
\hline 3 & 5 & 0.092256 & 0.001167 & 11.3 & 7 & 106 \\
\hline 4 & 6 & 0.011366 & 0.066176 & 12.9 & 6 & 98 \\
\hline 5 & 4 & 0.007456 & 0.002754 & 10.5 & 8 & 105 \\
\hline 6 & 7 & 0.008310 & 0.002895 & 15.2 & 10 & 113 \\
\hline 7 & 8 & 0.082839 & 0.002895 & 20.1 & 4 & 89 \\
\hline 8 & 4 & 0.008356 & 0.012486 & 12.3 & 7 & 143 \\
\hline 9 & 5 & 0.010455 & 0.003874 & 10.8 & 6 & 123 \\
\hline 10 & 3 & 0.007234 & 0.042158 & 7.8 & 6 & 136 \\
\hline
\end{tabular}

\subsection{WSNs Performance Evaluation Process}

Simulation was carried out by putting the pre-processed data into the selforganization feature mapping network. Grid scale was $8 \times 4$, and the training step was 900. The integrated performance grade of each WSN was calculated by simulation. Variable $\xi$ reflects boundary characteristics of the WSNs performance level of each network. According to its values to cope, variable $\xi$ can be divided into five intervals. Lowest-level (I) $\xi<2.50$; Lower-level (II), $2.50<\xi \quad<3.10$; Moderate-level (III): $3.1<\xi$ $<3.50$; High-level (IV): $3.50<\xi<4.0$; Upper most-level (V): $\xi>4.0$. The simulation results of network performance grade for each WSN concert are shown in Table 2.

Table 2. ६ Simulation Values and Performance Grades of Various WSNs

\begin{tabular}{|c|c|c|}
\hline Number of WSNs & Value of $\xi$ & performance grade \\
\hline 1 & 2.15 & I \\
\hline 2 & 2.76 & II \\
\hline 3 & 3.45 & III \\
\hline 4 & 2.16 & IV \\
\hline 5 & 4.05 & V \\
\hline 6 & 3.26 & IV \\
\hline
\end{tabular}




\begin{tabular}{|c|c|c|}
\hline 7 & 4.54 & $\mathrm{~V}$ \\
\hline 8 & 2.56 & $\mathrm{I}$ \\
\hline 9 & 2.83 & II \\
\hline 10 & 3.05 & II \\
\hline
\end{tabular}

\subsection{Analysis of the Results}

From Table 2, the complex WSNs performance is closely related with factors such as accountability, agility, cost, actual performance, security and privacy. The performance grade distribution is distinct one by one. The upper-most-great level distribute in WSNs 4, WSNs6, WSNs 7. In WSNs 4, packet loss occurred frequently so that the actual performance level is low. Although its low actual performance level has partly offset accountability and agility, the integrated network performance level is still high. In WSNs 4, because the accountability, agility, and cost are low, and the cost level is high, the integrated network performance level is still as low as WSNs 1. In order to analyze the interaction of components, contacting weight values of indices are selected from the system model in Table 3.

Table 3. Contacting Weight Values of Indices

\begin{tabular}{|c|c|c|c|c|c|}
\hline $\begin{array}{c}\text { Number of } \\
\text { WSNs }\end{array}$ & $\begin{array}{c}\text { Accountability } \\
\left(x_{1}\right)\end{array}$ & $\begin{array}{c}\text { Actual } \\
\text { performance }\left(x_{2}\right)\end{array}$ & Agility $\left(x_{3}\right)$ & $\begin{array}{c}\text { Security and } \\
\text { privacy }\left(x_{4}\right)\end{array}$ & Cost $\left(x_{5}\right)$ \\
\hline 1 & 0.683 & 0.523 & 0.236 & 0.147 & 0.684 \\
\hline 2 & 0.542 & 0.178 & 0.565 & 0.621 & 0.268 \\
\hline 3 & 0.126 & 0.283 & 0.268 & 0.874 & 0.806 \\
\hline 4 & 0.365 & 0.176 & 0.743 & 0.284 & 0.306 \\
\hline 5 & 0.518 & 0.376 & 0.368 & 0.596 & 0.589 \\
\hline 6 & 0.256 & 0.114 & 0.129 & 0.478 & 0.136 \\
\hline 7 & 0.572 & 0.268 & 0.348 & 0.589 & 0.689 \\
\hline 8 & 0.654 & 0.117 & 0.118 & 0.369 & 0.657 \\
\hline 9 & 0.629 & 0.687 & 0.159 & 0.746 & 0.258 \\
\hline 10 & 0.684 & 0.634 & 0.387 & 0.865 & 0.854 \\
\hline
\end{tabular}

A non-linear information dynamics model has explicit physical meanings, and is applied to evaluate WSNs performance of some samples, and results analysis shows its precision and efficiency. Meanwhile, our model also gives us some meaning conclusion, indexes have influence on each other, and they have contacting weight values between each other. Each component of WSNs performance index has a great contribution to the evaluation model. 


\section{Conclusion}

In recent years, with the further development and more applications emerging, WSNs is no longer confined to the traditional area. Based on the analysis mentioned above, we can obtain the overall network performance, and then evaluate the identification and acceptance of an existing network. As for a planned network, we also greatly depend on the evaluation of the scheme using the performance of WSNs, in order to choose the suitable network structure and the deployment of network nodes. Because WSNs is dynamic, distribution, resource constrained, uncertainty, complexity and heterogeneity, meanwhile different WSNs applications have diverse characteristics and requirements in the respective field, which both urge us to further research, especially performance evaluation of WSNs. In this paper, we expand the range of performance index which exceeds time delay, packet loss rate, and throughput. Meanwhile put forward a non-equilibrium statistical mechanics model for integrated WSNs performance evaluation from an evolutionary perspective. A non-linear information dynamics mode is introduced based on the maximum flux principle during modeling process. The integrated performance evaluation of WSNs can be achieved by giving reasonable weights of each evaluation index to stabilize the system. The new model reveals the formation pattern and the dynamics law of evolution we can analyze the interaction of components, contacting weight values of indices. Then, the proposed method has been used in complex simulation, most-great level distribute in WSNs 4, WSNs6, WSNs 7, and the results have indicated that the model is objective and effective. In the future, we will extend our ranking algorithm to cope with variation in performance attributes such as adopting fuzzy sets, neural network and so on.

\section{Acknowledgments}

"A Project Supported by Scientific Research Fund of Zhejiang Provincial Education Department (Y201119684)".

\section{References}

[1] Z. Ma, Y. Sun, and T. Mei, "Survey on wireless sensors network", JOURNAL-CHINA INSTITUTE OF COMMUNICATIONS, vol. 25, no. 4, (2004), pp. 114-124.

[2] I. D. Schizas, A. Ribeiro and G. B. Giannakis, "Consensus in ad hoc WSNs with noisy links-Part I: Distributed estimation of deterministic signals", Signal Processing, IEEE Transactions on vol. 56, no. 1, (2008), pp. 350-364.

[3] M. Keshtgari and A. Deljoo, "A Wireless Sensor Network Solution for Precision Agriculture Based on ZigBee Technology", Wireless Sensor Network, vol. 4, no. 1, (2012).

[4] Pereira and S. Silva, "Mean square convergence of consensus algorithms in random WSNs", Signal Processing, IEEE Transactions on vol. 58, no. 5, (2010), pp. 2866-2874.

[5] D. A. Swyt, "an Assessment of the United States Measurement System: Addressing Measurement Barriers to Accelerate Innovation", 2007 NIST Special Publication1048.

[6] W. M. Healy and W. S. Jang, "Practical Challenges in Wireless Sensor Network Use in Buildings", 2008 NIST Technical Note 1604.

[7] D. Karaboga, S. Okdem and C. Ozturk, "Cluster based wireless sensor network routing using artificial bee colony algorithm", Wireless Networks, vol. 18, no. 7, (2012), pp. 847-860.

[8] J. Yick, B. Mukherjee and D. Ghosal, "Wireless sensor network survey", Computer networks, vol. 52, no. 12, (2008), pp. 2292-2330.

[9] C. Huang and D. Du, "New constructions on broadcast encryption and key pre-distribution schemes", In: Proceedings of IEEE INFOCOM05, Miami: IEEE Press; (2005), p. 515-23.

[10] I. D. Schizas, A. Ribeiro and G. B. Giannakis, "Consensus in ad hoc WSNs with noisy links-Part I: Distributed estimation of deterministic signals", Signal Processing, IEEE Transactions on vol. 56, no. 1, (2008), pp. 350-364. 
[11] A. Mpitziopoulos, “A survey on jamming attacks and countermeasures in WSNs”, Communications Surveys \& Tutorials, IEEE vol. 11, no. 4, (2009), pp. 42-56.

[12] E. Stavrou and A. Pitsillides, "A survey on secure multipath routing protocols in WSNs", Computer Networks vol. 54, no. 13, (2010), pp. 2215-2238.

[13] P. Wright, D. Dornfeld and N. Ota, "Condition monitoring in end-milling using wireless sensor networks (WSNs)", Transactions of NAMRI/SME36 (2008), pp. 177-183.

[14] J. Deng, R. Han and S. Mishra, "A performance evaluation of intrusion tolerant routing in wireless sensor networks", Information Processing in Sensor Networks, Springer Berlin Heidelberg, (2003).

[15] D. Wang, "Building Wireless Sensor Networks (WSNs) by Zigbee Technology [J]", Journal of Chongqing university (natural science edition) vol. 8, (2006), p. 023.

[16] N. Wang, N. Zhang and M. Wang, "Wireless sensors in agriculture and food industry-Recent development and future perspective", Computers and electronics in agriculture, vol. 50, no. 1, (2006), pp. 1-14.

[17] V. C. Gungor, B. Lu and G. P. Hancke, "Opportunities and challenges of wireless sensor networks in smart grid”, Industrial Electronics, IEEE Transactions on vol. 57, no. 10, (2010), pp. 3557-3564.

[18] C.-F. Huang and Y.-C. Tseng, "The coverage problem in a wireless sensor network", Mobile Networks and Applications, vol. 10, no. 4, (2005), pp. 519-528.

[19] M. Gholami, N. Cai and R. W. Brennan, "An artificial neural network approach to the problem of wireless sensors network localization”, Robotics and Computer-Integrated Manufacturing, vol. 29, no. 1, (2013), pp. 96-109.

[20] S. Okdem, D. Karaboga and C. Ozturk, "An application of wireless sensor network routing based on artificial bee colony algorithm", Evolutionary Computation (CEC), 2011 IEEE Congress on. IEEE, (2011).

[21] B. Bhuyan, "Quality of Service (QoS) Provisions in Wireless Sensor Networks and Related Challenges", Wireless Sensor Network, vol. 2, no. 11, (2010).

[22] M. Dohler, "The ARESA project: Facilitating research, development and commercialization of WSNs. Sensor", Mesh and Ad Hoc Communications and Networks, 2007. SECON07. 4th Annual IEEE Communications Society Conference on. IEEE, (2007).

[23] L. Ravindranath, "Improving wireless network performance using sensor hints", Proceedings of the 8th USENIX conference on Networked systems design and implementation. USENIX Association, (2011).

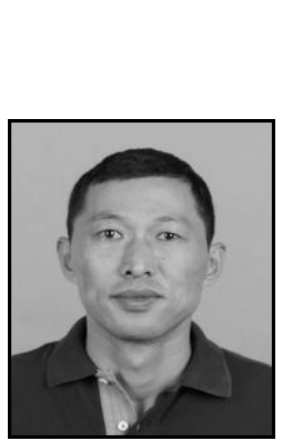

\section{Author}

Ye Chen, He received his M.Sc. in Mathematics (1977) and $\mathrm{PhD}$ in Information Sciences (2002) from University. Now he is full professor of informatics at Mathematics-Informatics Department, University. Since 2012 he is rector of University. Since 2011 he is Senior Member of IEEE. His current research interests include different aspects of Artificial Intelligence and Distributed Systems. 